\title{
Protective effect of berberine on acute cardiomyopathy associated with doxorubicin treatment
}

\author{
CHEN XIONG $^{1,2}$, YAN-ZHAO WU ${ }^{3}$, YU ZHANG $^{4}$, ZI-XIAO WU $^{1,2}$, XUE-YAN CHEN $^{1,2}$, \\ PING JIANG ${ }^{1,2}$, HUI-CAI GUO ${ }^{1,2}$, KE-RANG XIE ${ }^{1,2}$, KE-XIN WANG $^{1,2}$ and SU-WEN SU ${ }^{1,2}$

\begin{abstract}
${ }^{1}$ Key Laboratory of Neural and Vascular Biology, Ministry of Education; ${ }^{2}$ Key Laboratory of Pharmacology and
${ }^{3}$ Department of Otorhinolaryngology-Head and Neck Surgery, The Fourth Hospital of Hebei Medical University, Shijiazhuang, Hebei 050011; ${ }^{4}$ Center for Reproductive Medicine, Family Planning Scientific and Technical Institution of Hebei Province, Shijiazhuang, Hebei 050000, P.R. China
\end{abstract} \\ Toxicology for New Drugs, Department of Pharmacology, Hebei Medical University, Shijiazhuang, Hebei 050017;
}

Received June 27, 2017; Accepted December 22, 2017

DOI: $10.3892 / \mathrm{ol} .2018 .8020$

\begin{abstract}
Doxorubicin (DOX) is a potent and broad-spectrum anthracycline chemotherapeutic agent, but dose-dependent cardiotoxic side effects limit its clinical application. This toxicity is closely associated with the generation of reactive oxygen species (ROS) radical during DOX metabolism. The present study investigated the effects of Berberine (Ber) on DOX-induced acute cardiac injury in a rat model and analysed its mechanism in cardiomyocytes in vitro. Serum creatine kinase $(\mathrm{CK})$, creatine kinase isoenzyme (CK-MB) and malondialdehyde (MDA) levels were significantly increased in the DOX group compared with the control group. This increase was accompanied by cardiac histopathological injury and a decrease in cardiomyocyte superoxide dismutase (SOD) and catalase (CAT). CK, CK-MB and MDA levels decreased and SOD and CAT levels increased in the Ber-treated group compared to the DOX group. Ber ameliorated the DOX-induced increase in cytosolic calcium concentration $\left(\left[\mathrm{Ca}^{2+}\right]_{\mathrm{i}}\right)$, attenuated mitochondrial $\mathrm{Ca}^{2+}$ overload and restored the DOX-induced loss of mitochondrial membrane potential in vitro. These results demonstrated that Ber exhibited protective effects against DOX-induced heart tissue free radical injury, potentially via the inhibition of intracellular $\mathrm{Ca}^{2+}$ elevation and attenuation of mitochondrial dysfunction.
\end{abstract}

Correspondence to: $\mathrm{Dr}$ Su-Wen Su, Key Laboratory of Pharmacology and Toxicology for New Drugs, Department of Pharmacology, Hebei Medical University, 361 East Zhongshan Road, Shijiazhuang, Hebei 050017, P.R. China

E-mail: suswmk@hebmu.edu.cn

Abbreviations: DOX, doxorubicin; Ber, berberine; ROS, reactive oxygen radical species; $\Delta \Psi_{\mathrm{m}}$, mitochondrial membrane potential; $\mathrm{CK}$, creatine kinase; CK-MB, creatine kinase isoenzyme; MDA, malondialdehyde; SOD, superoxide dismutase; CAT, catalase

Key words: berberine, cardiotoxicity, doxorubicin, mitochondrial, cytosolic calcium concentration, ventricular myocytes

\section{Introduction}

Doxorubicin (DOX) is an effective chemotherapeutic agent that is widely used to treat numerous types of neoplasms. However, serious cardiotoxic side effects, which may cause arrhythmia and heart failure, limit its clinical application (1-4). Multiple mechanisms are involved in DOX cardiotoxicity, including lipid peroxidation and decreased glutathione levels (5), calcium overloading and mitochondrial dysfunction via increases in mitochondrial calcium and the generation of reactive oxygen species (ROS) $(6,7)$. Increased oxidative stress and an antioxidant deficit serve key roles in DOX-induced cardiotoxicity. Previous data suggests that the dysregulation of calcium handling and mitochondrial function contribute to DOX-induced cardiotoxicity (8). Multiple cardioprotective drugs may be combined to eliminate its cardiotoxicity or reduce doses to an acceptable level are expected to increase its efficacy (8). The majority of these attempts produced beneficial effects, but the search for more effective strategies against DOX-induced complications achieved little success (6). Therefore, additional adjuvant drugs are co-administered with DOX to patients with neoplastic diseases to reduce DOX-induced cardiotoxicity or enhance its therapeutic effects (8).

Berberine (Ber) is an isoquinoline alkaloid that was originally extracted from the traditional Chinese plant Coptis chinensis (Huang Lian), and is an established treatment for diarrhoea in traditional Chinese medicine (9). Ber exhibits a wide range of pharmacological activities, including: Antioxidant properties to attenuate reactive oxygen species (ROS) formation in various tissues; anti-diabetic; anti-hyperlipidaemic; anti-inflammatory; anti-tumour; and cardio-protective effects $(9,10)$. We hypothesized that the antioxidant and cardio-protective effects of Ber may exhibit protective effects against DOX-induced cardiomyopathy. The present experimental study investigated the possible protective effects of Ber against acute DOX-induced cardiotoxicity induced in a rat model. The effect of Ber on changes in known indicators of cardiotoxicity and oxidative stress including serum creatine kinase $(\mathrm{CK})$, creatine kinase isoenzyme 
(CK-MB) activities and serum and myocardial superoxide dismutase (SOD), malondialdehyde (MDA) and catalase (CAT) contents was investigated. The present study may support the utility of Ber as a safe, clinically-approved drug in the treatment of cancer.

\section{Materials and methods}

Drugs and chemicals. DOX was provided by Lingnan Pharmaceutical, Ltd. (Guangzhou, China). Ber was provided by Acros Organics (Geel, Belgium). Fluo3-AM was purchased from Sigma-Aldrich; Merck KGaA (Darmstadt, Germany). MDA, CAT and SOD assay kits were purchased from Nanjing Jiancheng Bioengineering Institute (Nanjing, China). CK and CK-MB assay kits were purchased from Sysmex Corporation (Kobe, Japan). Rhodamine (Rh-123) and Rhod-2-acetoxymethyl (rhod-2-AM) were purchased from Molecular Probes; Thermo Fisher Scientific, Inc., (Waltham, MA, USA). The working solutions of Harris Haematoxylin and eosin were purchased from Baso Diagnostics Inc. Zhuhai (New Taipei City, Taiwan).

Animals and treatments. All experiments were performed in compliance with the Guide for the Care and Use of Laboratory Animals (11) and were reviewed and approved by the Ethics Committee for the Use of Experimental Animals at Hebei Medical University (Shijiazhuang, China). Sprague-Dawley (SD) rats weighing 200-250 g were obtained from the medical laboratory of Hebei Medical University. The animals were acclimated to the laboratory environment for 1 week in standard experimental conditions ( $12 \mathrm{~h}$ light:12 $\mathrm{h}$ dark schedule) and allowed access to food and water ad libitum. Animal experiments were performed in accordance with the National Institutes of Health guidelines for the experimental use of animals (11). Rats were randomly assigned to the following five groups of 10 animals each: Water-treated (control) group, DOX-treated group and DOX plus Ber treatment at doses of 5,10 and $20 \mathrm{mg} / \mathrm{kg}$. The control and DOX groups received distilled water orally for 10 consecutive days. Ber was administered at the aforementioned doses orally once daily for 10 consecutive days. The dosing volume was $1 \mathrm{ml} / 100 \mathrm{~g}$ body weight. All rats were intraperitoneally injected with a single dose of DOX $20 \mathrm{mg} / \mathrm{kg}$ on day 8 , with the exception of the control group. The selected dose was based on our previous study (12).

Sample collection and biochemical assays. All rats were anaesthetized $48 \mathrm{~h}$ after DOX injection, and blood samples were collected prior to sacrifice. Serum was separated for CK and CK-MB assays. The heart was quickly isolated, blotted dry on filter paper, and weighed. The heart was cut and prepared for histopathological examination, and one part was prepared as $10 \%$ homogenates in ice-cold saline for the determination of MDA, CAT activity and SOD contents. The total protein content was detected with a bicinchoninic acid protein Assay kit (Pierce, Thermo Fisher Scientific Inc.), and the activities of MDA, CAT and SOD in the cardiac tissue were expressed as units/mg protein.

Histopathological examinations. To analyse the histopathologic changes of the cardiac tissue, one part of the heart was fixed in $10 \%$ buffered formalin, embedded in paraffin and dehydrated in an ascending series of ethanol (70, 80, 96, and $100 \%$ ). Tissue samples were embedded in paraffin and cut into $5-\mu \mathrm{m}$ thick slices. The sections were stained at room temperature with haematoxylin working solution and $1 \%$ eosin $(\mathrm{H} \& \mathrm{E})$ for 2 min respectively for histological analysis under a light microscope (magnification, x200, Olympus BX-50; Olympus Corporation, Tokyo, Japan).

General toxicity observation. A total of 10 animals were used to determine mortality in each group. The humane endpoints were a weight loss above $15 \%$ of initial weight or animal in a state of prostration. General conditions, mortality and body weight of the animals were observed daily until the end of the experiment. Fluid accumulation in the abdominal cavity was assessed at the end of the experiment subsequent to abdominal opening and scored on a graded scale of 0 to $3^{+}$, where: 0 , none; $1^{+}$, mild; $2^{+}$, moderate; and $3^{+}$, severe (13).

Acute isolation of cardiac myocytes. Single cardiac myocytes were enzymatically isolated from adult male rat hearts as described previously (14). Briefly, SD rats (200-250 g) were anaesthetized using pentobarbital sodium $(50 \mathrm{mg} / \mathrm{kg}$, intraperitoneally). The hearts were rapidly excised, mounted through the aorta, and perfused on a modified Langendorff apparatus with a calcium-free Tyrode solution at $37^{\circ} \mathrm{C}$, followed by $0.6 \%$ collagenase II in a Ca-free Tyrode solution to digest the heart. Isolated ventricular myocytes were maintained in Krebs solution. All experiments were performed within $6 \mathrm{~h}$ of ventricular myocyte isolation.

Measurement of cytosolic calcium concentration $\left[\mathrm{Ca}^{2+}\right]_{i}$. Isolated myocytes were prepared as aforementioned and loaded with $20 \mu \mathrm{mol} / 1$ membrane-permeable Fluo3-AM working solution containing $0.03 \%$ pluronic $\mathrm{F}-127$ at $37^{\circ} \mathrm{C}$ for $60 \mathrm{~min}$. The cells were superfused with fresh Tyrode solution three times at $25^{\circ} \mathrm{C}$ to allow de-esterification of Fluo3-AM. Cells were mounted in a chamber on the stage of an inverted microscope. Only cells with a rod shape and visible striations were used. Fluo3-AM fluorescence in cells was excited at $488 \mathrm{~nm}$, and fluorescence emission was recorded at $530 \mathrm{~nm}$ using a photomultiplier. The fluorescence signal was detected using a confocal laser scanning system (Leica TCS-SP II; Leica Microsystems GmbH, Germany). Calcium measurement is represented as relative fluorescence intensity $\left(\left(\mathrm{FI}-\mathrm{FI}_{0}\right) / \mathrm{FI}_{0}\right.$, \%; $\mathrm{FI}_{0}$ : Control; FI: Administration of drugs). Cells were perfused with normal Tyrode solution for 2 min and perfused with Tyrode solution containing Ber for $15 \mathrm{~min}$. A total of 50-100 images were scanned from each cell, and the data were analysed by a confocal laser microscopic system (Leica TCS SP2, Mannheim, Germany).

Mitochondrial $\mathrm{Ca}^{2+}$ concentration and membrane potential measurements. The mitochondrial membrane potential $\left(\Delta \Psi_{\mathrm{m}}\right)$ was visualized in cardiomyocytes stained with the fluorescent probe Rh-123. Myocytes were incubated with $0.5 \mu \mathrm{M}$ Rh-123 for $10 \mathrm{~min}$ at $37^{\circ} \mathrm{C}$. Rh-123 fluorescence was detected at the emission wavelength of $490 \mathrm{~nm}$, and the green emission fluorescence was excited at $530 \mathrm{~nm}$ to analyse $\Delta \Psi_{\mathrm{m}}$. The mitochondrial $\mathrm{Ca}^{2+}$ concentration $\left[\left(\mathrm{Ca}^{2+}\right)_{\mathrm{m}}\right]$ was measured using the 
Table I. Effect of Ber on DOX-induced abdominal, pleural and pericardial effusion intensity scores in surviving rats.

\begin{tabular}{|c|c|c|c|c|c|c|c|c|c|}
\hline \multirow[b]{3}{*}{ Group } & \multirow[b]{3}{*}{$\mathrm{n}$} & \multicolumn{8}{|c|}{ Effusion intensity score } \\
\hline & & \multicolumn{2}{|c|}{0} & \multicolumn{2}{|c|}{+} & \multicolumn{2}{|c|}{++} & \multicolumn{2}{|c|}{+++} \\
\hline & & $\mathrm{N}$ & $\%$ & $\mathrm{~N}$ & $\%$ & $\mathrm{~N}$ & $\%$ & $\mathrm{~N}$ & $\%$ \\
\hline Con & 10 & 10 & 100 & 0 & 0 & 0 & 0 & 0 & 0 \\
\hline DOX & 8 & 0 & 0 & 0 & 0 & 0 & 0 & 8 & $100^{\mathrm{a}}$ \\
\hline $\operatorname{Ber}(5 \mathrm{mg} / \mathrm{kg})$ & 10 & 0 & 0 & 4 & 40 & 3 & 30 & 3 & $30^{\mathrm{a}, \mathrm{b}}$ \\
\hline Ber $(10$ mg/kg) & 10 & 1 & 10 & 4 & 40 & 2 & 20 & 3 & $30^{a, b}$ \\
\hline $\operatorname{Ber}(20$ mg/kg) & 10 & 1 & 10 & 5 & 50 & 2 & 33.3 & 2 & $20^{\mathrm{a}, \mathrm{b}}$ \\
\hline
\end{tabular}

Con, control; DOX, doxorubicin; Ber, berberine. 0, none; + , mild; ++ , moderate; +++ , severe. Statistical evaluation was performed using the $\chi^{2}$-test. ${ }^{\mathrm{a}} \mathrm{P}<0.01$ vs. Con. ${ }^{\mathrm{b}} \mathrm{P}<0.01$ vs. $\mathrm{DOX}$.

$\mathrm{Ca}^{2+}$-sensing dye rhod-2-AM in loaded cells. Myocytes were exposed to $10 \mu \mathrm{M}$ rhod-2 acetoxymethyl ester for $120 \mathrm{~min}$ at $4^{\circ} \mathrm{C}$, and transferred to $37^{\circ} \mathrm{C}$ for an additional $30 \mathrm{~min}$ in the Tyrode solution (14). Rhod-2 fluorescence was excited via excitation at $540 \mathrm{~nm}$, and a $570 \mathrm{~nm}$ bandpass barrier filter was used to analyse $\left[\mathrm{Ca}^{2+}\right]_{\mathrm{m}}$. The fluorescence signal was detected using a confocal laser scanning system mentioned before. Regions of rod-shaped cardiomyocytes were outlined/highlighted to represent changes in fluorescence intensity over time, and the intensity of the selected region of the image was analysed using a confocal laser microscopic system. The fluorescence intensity was normalized to the initial value $\left(\mathrm{F}_{0}\right)$ recorded in normal Tyrode solution in each experiment, and the density ratio represented the relative fluorescence: $\left(\mathrm{FI}-\mathrm{FI}_{0}\right) / \mathrm{FI}_{0}$ $\mathrm{x} 100$, where $\mathrm{FI}_{0}$ represents the control and FI represents the drug-treated groups. Rat cardiomyocytes were treated with 0.1 or $1 \mu \mathrm{M}$ Ber for $20 \mathrm{~min}$ and exposed to $1 \mu \mathrm{M}$ DOX for $15 \mathrm{~min}$, which is the minimum time required for effective changes in $\left[\mathrm{Ca}^{2+}\right]_{\mathrm{m}}$ and $\Delta \Psi_{\mathrm{m}}(15)$.

Statistical analysis. All data are presented as the mean \pm standard deviation. Statistical tests were conducted using Microsoft Excel 2010 (version number, 14.0.4760.1000; Microsoft Corporation, Redmond, WA, USA) and SPSS for windows (version 11.0; SPSS Inc., Chicago, IL, USA). Following a one-way analysis of variance, quantitative data were analysed with post hoc contrasts by Fisher's least significant difference test. A chi-squared test was used to analyse the difference of the injury severity scores of the heart among the various treatment groups. $\mathrm{P}<0.05$ was considered to indicate a statistically significant difference.

\section{Results}

Effects of Ber on animal body weight and survival in DOX-induced cardiotoxicity. DOX treatment for two days reduced rat body weight compared with the control group ( $235 \pm 35$ vs. $266 \pm 15 \mathrm{~g} ; \mathrm{P}<0.01)$. Treatment with 5, 10 and $20 \mathrm{mg} / \mathrm{kg}$ Ber exerted no marked increment effects on body weight $(231 \pm 36,226 \pm 16$ and $253 \pm 54$ g, respectively; $P>0.05$ vs. DOX alone). There was no significant difference in the heart weight/body weight ratio between the five groups $(3.0 \pm 0.4 \mathrm{mg} / \mathrm{g}$ in the control group vs. $3.1 \pm 0.3 \mathrm{mg} / \mathrm{g}$ in DOX treatment and $2.8 \pm 0.5,3.3 \pm 0.3$ and $2.9 \pm 0.3 \mathrm{mg} / \mathrm{g}$ in the Ber 5,10 , and $20 \mathrm{mg} / \mathrm{kg}$ groups, respectively).

Effects of Ber on general toxicity in DOX-induced cardiotoxicity. A total of 2 rats in the DOX-only treated group succumbed 2 days following DOX injection. No mortality was observed in the Ber+DOX-treated groups. All surviving rats in the DOX-only treated group appeared weak, with scruffy fur and developed a light-yellow tinge. These animals exhibited a significant decrease in body weight compared to the Ber+DOX-treated groups. Notably, these animals developed congestive heart failure, which manifested as marked ascites during necropsy. The hallmark gross pathological changes in DOX-only treated rats were excessive amounts of pericardial, pleural and peritoneal fluids. The maximum ascites volume exhibited by a single animal in DOX-only treated group was $11.5 \mathrm{ml}$. Compared with DOX alone $(2.9 \pm 1.8 \%)$, the percentage of mean maximum ascites volume to animal body weight were decreased obviously in 5, 10 and $20 \mathrm{mg} / \mathrm{kg}$ Ber+DOX-treated groups $(1.8 \pm 0.9,1.0 \pm 0.1$ and $1.4 \pm 1.1 \%$ : $\mathrm{P}<0.05$, respectively). The effusion intensity score was severe in $100 \%$ of the DOX-only treated animals compared with 20-30\% severe scores in the Ber+DOX groups (Table I).

Ber alleviates DOX-induced oxidative damage. Serum CK-MB and CK levels are widely used as clinical markers for oxidative stress, which indicate myocardial injury (Table II). Oxidative stress was evident in the cardiac myocytes of the DOX groups, with significant increases in serum $\mathrm{CK}$ and $\mathrm{CK}-\mathrm{MB}$ levels compared with the control $(\mathrm{P}<0.01)$, and Ber treatment attenuated these increases in a dose-dependent manner $(\mathrm{P}<0.05)$. CK and CK-MB values in the Ber 10 and $20 \mathrm{mg} / \mathrm{kg}$ groups were almost equivalent to control levels. Serum and heart tissues of DOX-only treated rats revealed a significant increase in MDA and decreased CAT and SOD contents compared to the control group. MDA, CAT and SOD concentrations recovered markedly in the Ber+DOX groups compared to the DOX-only group. However, $5 \mathrm{mg} / \mathrm{kg}$ Ber did not prevent the DOX-induced increase in cardiac enzymes (Tables II and III). 
Table II. Serum levels of CAT, SOD, MDA, CK, and CK-MB activities following acute DOX intoxication and protective activity by Ber.

\begin{tabular}{lrrlcrc}
\hline Group & $\mathrm{n}$ & CAT $(\mathrm{U} / \mathrm{ml})$ & SOD $(\mathrm{U} / \mathrm{ml})$ & MDA $(\mathrm{mmol} / \mathrm{ml})$ & CK $(\mathrm{U} / \mathrm{l})$ & CK-MB $(\mathrm{U} / \mathrm{l})$ \\
\hline Con & 10 & $29.72 \pm 11.68$ & $41.35 \pm 3.60$ & $40.42 \pm 6.73$ & $727 \pm 261$ & $1,843 \pm 659$ \\
DOX & 8 & $19.79 \pm 4.53^{\mathrm{a}}$ & $23.41 \pm 12.10^{\mathrm{b}}$ & $55.37 \pm 6.20^{\mathrm{b}}$ & $1,426 \pm 540^{\mathrm{b}}$ & $3,404 \pm 939^{\mathrm{b}}$ \\
Ber $(5 \mathrm{mg} / \mathrm{kg})$ & 10 & $28.53 \pm 7.72^{\mathrm{d}}$ & $36.44 \pm 9.65^{\mathrm{c}}$ & $46.93 \pm 7.90^{\mathrm{c}}$ & $1,069 \pm 366^{\mathrm{a}}$ & $2,694 \pm 571^{\mathrm{a}}$ \\
Ber $(10 \mathrm{mg} / \mathrm{kg})$ & 10 & $25.70 \pm 5.16^{\mathrm{c}}$ & $44.76 \pm 3.02^{\mathrm{a}, \mathrm{d}}$ & $47.98 \pm 8.81^{\mathrm{c}}$ & $991 \pm 271^{\mathrm{c}}$ & $2,395 \pm 618^{\mathrm{c}}$ \\
Ber $(20 \mathrm{mg} / \mathrm{kg})$ & 10 & $27.51 \pm 10.20^{\mathrm{c}}$ & $41.51 \pm 3.77^{\mathrm{d}}$ & $40.47 \pm 18.21^{\mathrm{c}}$ & $792 \pm 378^{\mathrm{c}}$ & $2,123 \pm 865^{\mathrm{c}}$ \\
\hline
\end{tabular}

Data are presented as mean \pm standard deviation. Con, control; DOX, doxorubicin; Ber, berberine; CAT, catalase; MDA, malondialdehyde; SOD, superoxide dismutase; CK, creatine kinase; CK-MB, creatine kinase isoenzyme. ${ }^{a} \mathrm{P}<0.05$ and ${ }^{b} \mathrm{P}<0.01$ vs. Con; ${ }^{\mathrm{C}} \mathrm{P}<0.05$ and ${ }^{\mathrm{d}} \mathrm{P}<0.01 \mathrm{vs}$. DOX.

Table III. Effect of Ber on peroxidative alterations induced by acute DOX intoxication in rat cardiac tissue.

\begin{tabular}{lcccc}
\hline Group & $\mathrm{n}$ & CAT (U/mg prot) & SOD (U/mg prot) & MDA (nmol/mg prot) \\
\hline Con & 10 & $52.0 \pm 4.5$ & $54.3 \pm 8.5$ & $28.73 \pm 3.94$ \\
DOX & 8 & $37.9 \pm 13.4^{\mathrm{b}}$ & $42.1 \pm 6.2^{\mathrm{b}}$ & $42.59 \pm 4.93^{\mathrm{b}}$ \\
Ber $(5 \mathrm{mg} / \mathrm{kg})$ & 10 & $48.3 \pm 9.9$ & $46.15 \pm 7.25^{\mathrm{a}}$ & $42.50 \pm 7.22^{\mathrm{b}}$ \\
Ber $(10 \mathrm{mg} / \mathrm{kg})$ & 10 & $52.6 \pm 1.7^{\mathrm{d}}$ & $49.32 \pm 7.12^{\mathrm{c}}$ & $35.41 \pm 5.38^{\mathrm{a}, \mathrm{d}}$ \\
Ber $(20 \mathrm{mg} / \mathrm{kg})$ & 10 & $52.9 \pm 2.6^{\mathrm{c}}$ & $50.97 \pm 6.77^{\mathrm{d}}$ & $33.00 \pm 1.15^{\mathrm{b}, \mathrm{d}}$ \\
\hline
\end{tabular}

Con, control; DOX, doxorubicin; Ber, berberine; CAT, catalase; MDA, malondialdehyde; SOD, superoxide dismutase; mg prot, mg protein. ${ }^{a} \mathrm{P}<0.05$ and ${ }^{\mathrm{b}} \mathrm{P}<0.01$ vs. Con; ${ }^{\mathrm{c}} \mathrm{P}<0.05$ and ${ }^{\mathrm{d}} \mathrm{P}<0.01$ vs. DOX. Data are presented as mean \pm standard deviation.

Histopathological examination. DOX-induced cardiotoxicity was additionally assessed using H\&E-stained sections. Heart tissue from the control groups exhibited regular cell distribution and normal myocardium architecture. Histological examination of cardiac sections using H\&E stain revealed that DOX induced cardiomyocyte cytoplasmic vacuolization, interstitial oedema and inflammatory cell infiltration. Myocardial lesions were attenuated in the Ber+DOX groups compared to the DOX-alone group (Figs. 1 and 2).

Effect of Ber on the DOX-induced increase in $\left[\mathrm{Ca}^{2+}\right]_{i} .\left[\mathrm{Ca}^{2+}\right]_{\mathrm{i}}$ is critically important in the contraction and relaxation of cardiomyocytes (14). DOX-induced alterations in $\mathrm{Ca}^{2+}$ homeostasis is one possible mechanism of cardiotoxicity (15). Therefore, the effect of DOX on $\left[\mathrm{Ca}^{2+}\right]_{\mathrm{i}}$ in isolated cardiomyocytes was examined using changes in the fluorescence intensity of cardiomyocytes loaded with Fluo-3 AM. Fig. 3A demonstrates the effects of $1 \mu \mathrm{M}$ DOX on the increase in basal Fluo-3 fluorescence in the presence or absence of $1 \mu \mathrm{M}$ Ber in rat cardiomyocytes. Treatment of cardiomyocytes with $1 \mu \mathrm{M}$ DOX markedly increased $\left[\mathrm{Ca}^{2+}\right]_{\mathrm{i}}$ levels, and the cardiomyocytes gradually became 'rounded' into a contracture state in 15 min (Fig. 3A-a). However, the cardiomyocytes treated with Ber did not become rounded (Fig. 3A-b). Fig. 3B summarizes the quantitative data. DOX $(1 \mu \mathrm{M})$ increased $\left[\mathrm{Ca}^{2+}\right]_{\mathrm{i}}$ levels to $186.6 \pm 18.3 \%$ at $5 \mathrm{~min}$ and $252.1 \pm 11.1 \%$ at $15 \mathrm{~min} \mathrm{DOX}$, and the extent of the increase in $\left[\mathrm{Ca}^{2+}\right]_{\mathrm{i}}$ at $15 \mathrm{~min}$ was significantly $>5$ min $(\mathrm{P}<0.05)$. Pre-treatment with $1 \mu \mathrm{M}$ Ber significantly attenuated the $1 \mu \mathrm{M}$ DOX-induced elevation of $\left[\mathrm{Ca}^{2+}\right]_{\mathrm{i}}$ to $150.6 \pm 4.7 \%$ at $5 \mathrm{~min}$ and $164.2 \pm 10.8 \%$ at $15 \mathrm{~min}$. These results demonstrated that DOX increased $\left[\mathrm{Ca}^{2+}\right]_{\mathrm{i}}$ and that Ber attenuated this increase.

Effects of Ber on DOX-induced $\Delta \Psi_{m}$. Mitochondria are involved in the maintenance of $\mathrm{Ca}^{2+}$ homeostasis primarily due to their capacity to buffer cytosolic $\mathrm{Ca}^{2+}(14,15) . \Delta \Psi_{\mathrm{m}}$ is the central factor that controls the accumulation of $\mathrm{Ca}^{2+}$ within the mitochondrial matrix, cell respiration and ATP synthesis (14). The measurement of $\Delta \Psi_{\mathrm{m}}$ is a powerful tool for evaluating mitochondrial damage using the fluorescent probe Rhodamine 123 (Rh-123). Mitochondrial fluorescence intensity correlates quantitatively with changes in $\Delta \Psi_{\mathrm{m}}$ (14). Therefore, the effects of DOX on $\Delta \Psi_{\mathrm{m}}$ in rat ventricular myocytes were examined by evaluating $\Delta \Psi_{\mathrm{m}}$ in cardiomyocytes using the Rh-123 staining assay. The corresponding changes in Rh-123 fluorescence intensity in cardiomyocytes were measured 5 and 15 min following perfusion of $1 \mu \mathrm{M}$ DOX. Fig. 4A-a indicates the confocal images of $\Delta \Psi_{\mathrm{m}}$ following cell treatment for $15 \mathrm{~min}$ with $1 \mu \mathrm{M}$ DOX. The results revealed that DOX induced a marked decrease in mitochondrial membrane potential. However, the pre-treatment of myocytes with $1 \mu \mathrm{M}$ Ber significantly restored the DOX-induced reduction in $\Delta \Psi_{\mathrm{m}}(\mathrm{P}<0.05$, Fig. 4B) and attenuated the DOX-induced decrease in Rh-123 fluorescence intensity (Fig. 4A-b). These results demonstrated that Ber prevented the DOX-induced loss of the mitochondrial membrane potential. 


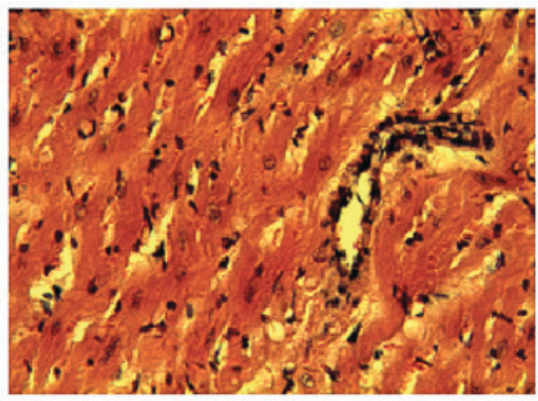

Con group

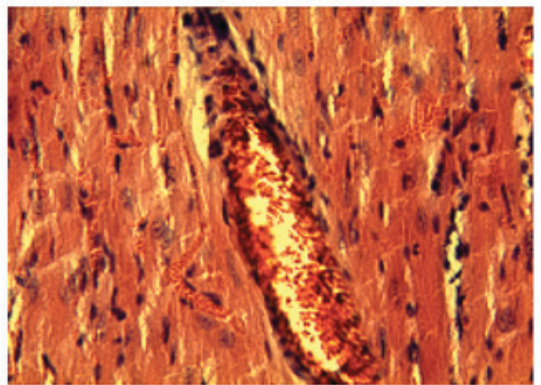

Ber $5 \mathrm{mg} / \mathrm{kg}$

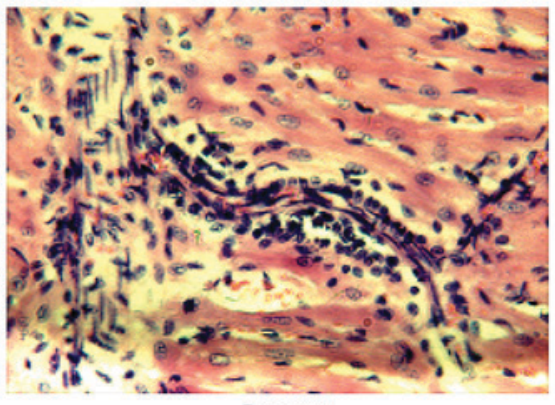

Dox group

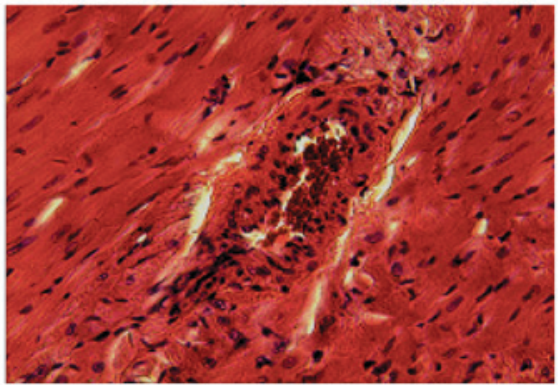

Ber $20 \mathrm{mg} / \mathrm{kg}$

Figure 1. Representative histopathological sections of haematoxylin and eosin staining of cardiac tissue in different groups. Marked interstitial oedema and inflammatory cells infiltration were observed in the DOX group. Interstitial oedema and inflammatory cell infiltration were alleviated in the Ber $5 \mathrm{mg} / \mathrm{kg}$ group. Minimal residual inflammatory cell infiltration was observed in the Ber $20 \mathrm{mg} / \mathrm{kg}$ group. (magnification, x200). DOX, doxorubicin; Ber, berberine; Con, normal cardiac tissue.

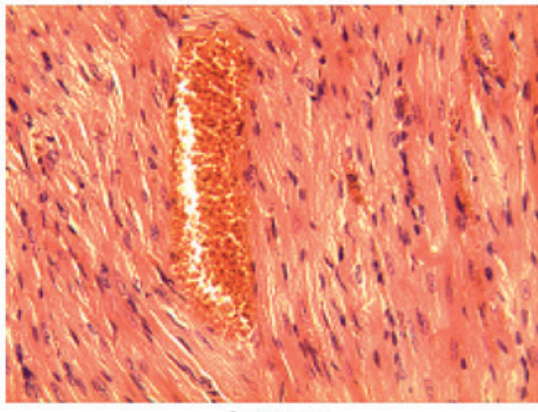

Con group

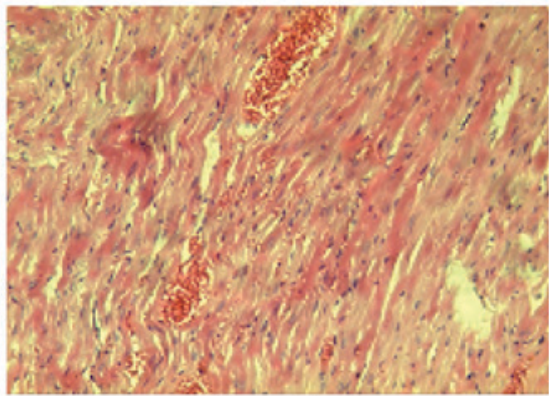

Ber $5 \mathrm{mg} / \mathrm{kg}$

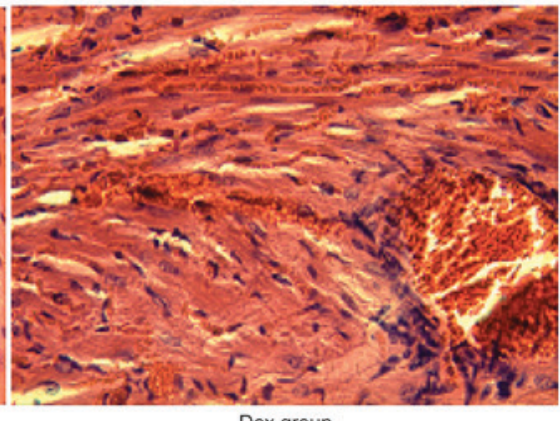

Dox group

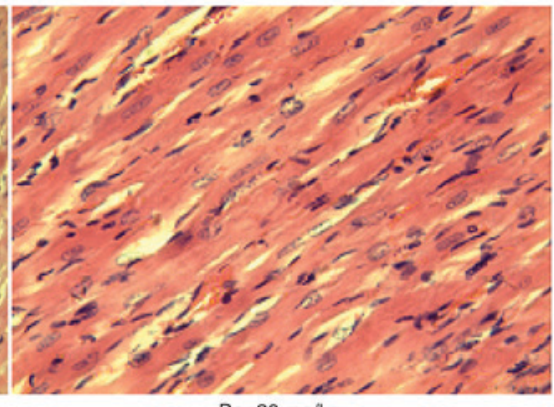

Ber $20 \mathrm{mg} / \mathrm{kg}$

Figure 2. Histological evaluation of haematoxylin and eosin staining of cardiac tissue from the untreated Dox and Ber-treated groups (magnification, x200). Normal cardiac structure was observed in the Con group. Bleeding and necrosis were observed in the interstitial cardiac tissues of the DOX group. Attenuated bleeding in cardiac tissues was observed in the Ber $5 \mathrm{mg} / \mathrm{kg}$ group. No notable bleeding in cardiac tissues was observed in the Ber $20 \mathrm{mg} / \mathrm{kg}$ group. DOX, doxorubicin; Ber, berberine; Con, normal cardiac tissue.

Effects of Ber on DOX-induced $\left[\mathrm{Ca}^{2+}\right]_{m}$ overload. $\mathrm{Ca}^{2+}$ overload in mitochondria may lead to mitochondrial dysfunction and be an important determinant of myocyte toxicity (14). Whether Ber attenuated the rise in DOX-induced $\left[\mathrm{Ca}^{2+}\right]_{\mathrm{m}}$ overload was investigated. Fig. 5A demonstrates confocal images of rat ventricular myocytes loaded with Rhod- 2 in the presence or absence of $1 \mu \mathrm{M}$ DOX or $1 \mu \mathrm{M}$ DOX $+1 \mu \mathrm{M}$ Ber. The resting $\left[\mathrm{Ca}^{2+}\right]_{\mathrm{m}}$ in intact cells was low, as revealed by the dim baseline signal of Rhod-2 fluorescence. Treatment with $1 \mu \mathrm{M}$ DOX significantly elevated $\left[\mathrm{Ca}^{2+}\right]_{\mathrm{m}}$ (Fig. 5A-a). Cells exhibited a high intensity of Rhod-2 fluorescence in the presence of $1 \mu \mathrm{M}$ DOX, and this fluorescence gradually increased. However, 


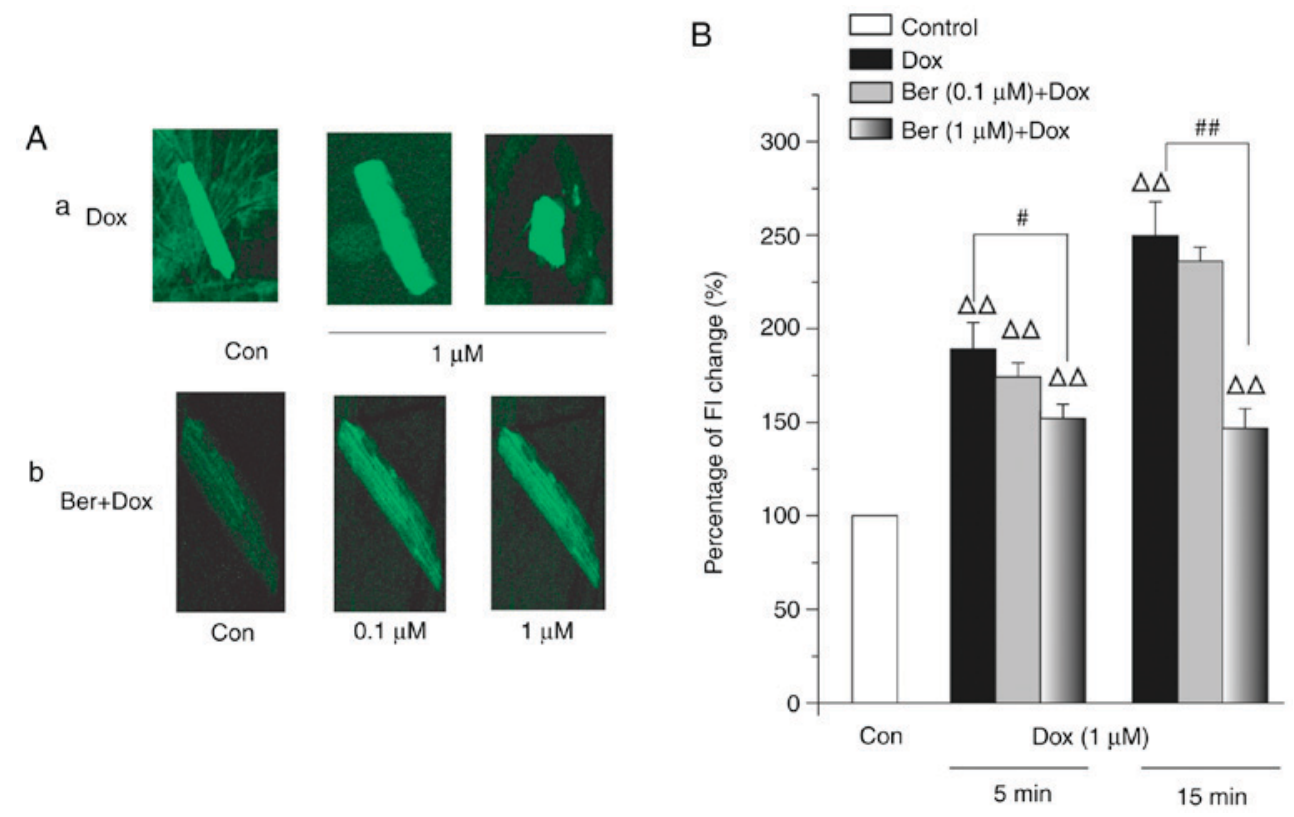

Figure 3. Effect of Ber on DOX-induced $\left[\mathrm{Ca}^{2+}\right]_{\mathrm{i}}$ elevation in isolated rat ventricular myocytes. (A) (a) Representative image of changes in fluorescence images of $\left[\mathrm{Ca}^{2+}\right]_{\mathrm{i}}$ in single isolated rat ventricular myocytes following Dox exposure, recorded at 15 min using laser confocal microscopy. (b) Effects of Ber incubation on DOX-induced $\left[\mathrm{Ca}^{2+}\right]_{\mathrm{i}}$ levels (magnification, $\mathrm{x} 40$ ). (B) Effects of Ber incubation on DOX-induced $\left[\mathrm{Ca}^{2+}\right]_{\mathrm{i}}$ elevation. Changes in $\left[\mathrm{Ca}^{2+}\right]_{\mathrm{i}}$ are represented as the ratio of $\mathrm{FI}-\mathrm{FI}_{0} / \mathrm{FI}_{0}$. ( $\mathrm{n}=7$ in each group) $\left({ }^{\Delta} \mathrm{P}<0.01\right.$ vs. control, ${ }^{\# \#} \mathrm{P}<0.01$ and $\left.{ }^{\#} \mathrm{P}<0.05\right)$. DOX, doxorubicin; Ber, berberine; con, control group; $\mathrm{FI}_{0}$, control; $\mathrm{FI}$, the drug-treated groups; $\left[\mathrm{Ca}^{2+}\right]_{\mathrm{i}}$, cytosolic calcium concentration.
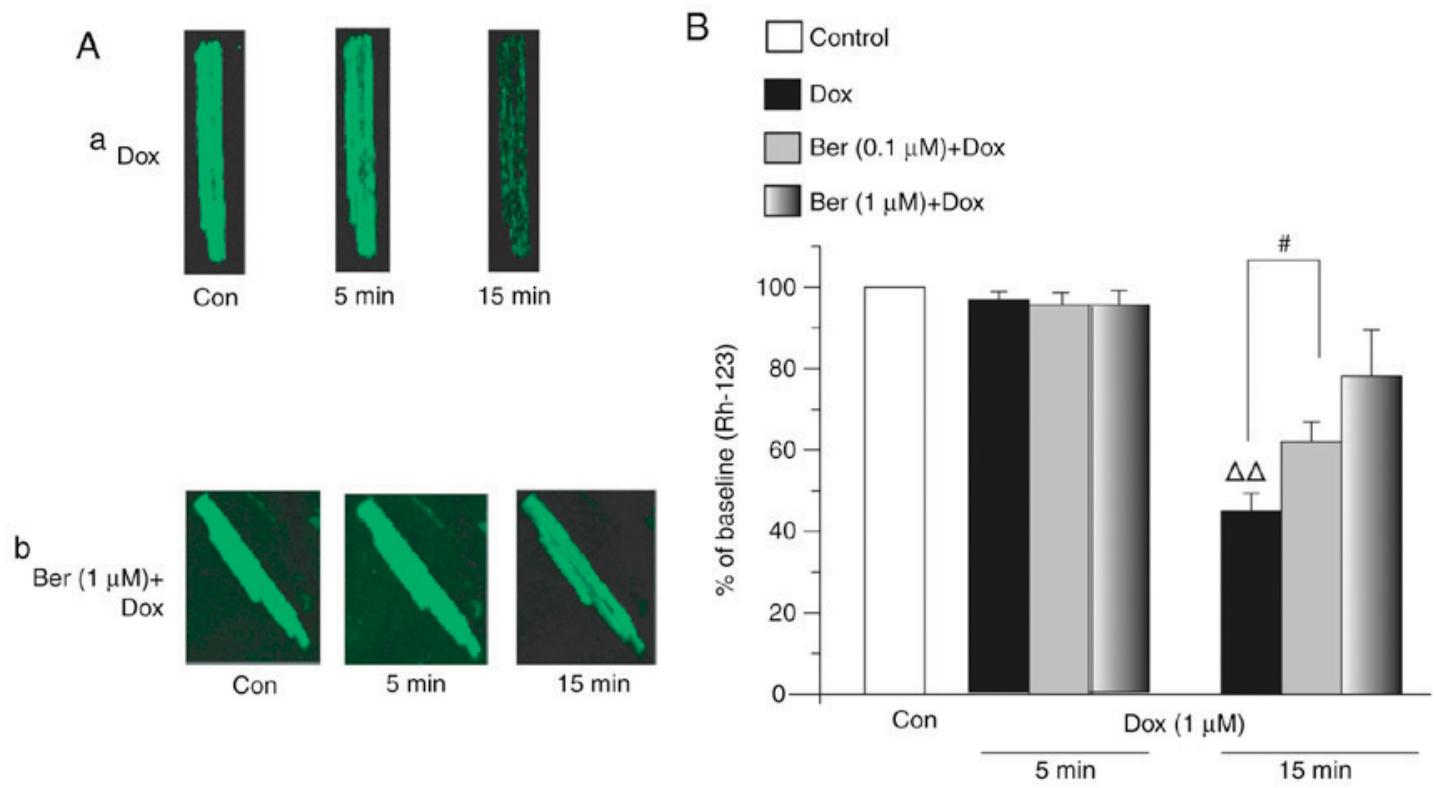

Figure 4. Effect of Ber on $\Delta \Psi \mathrm{m}$ in Rhodamine 123-loaded rat ventricular myocytes. (A) Confocal images of $\Delta \Psi_{\mathrm{m}}$ in single isolated rat ventricular myocytes. Cells were treated with DOX for 15 min as follows: (a) DOX and (b) Ber $(1 \mu \mathrm{M})+\mathrm{DOX}$ (magnification, $\mathrm{x} 40$ ). (B) Summary data of the relative changes of Rh-123 fluorescence measured 15 min prior (Con) and subsequent to drug treatment. Myocytes were pre-treated with different concentrations of Ber for $1 \mathrm{~h}$, exposed to $1 \mathrm{mM}$ DOX for $15 \mathrm{~min}$, and assayed for $\Delta \Psi_{\mathrm{m}}$. $\left(\mathrm{n}=6\right.$ in each group) $\left({ }^{\Delta \Delta} \mathrm{P}<0.01 \mathrm{vs}\right.$. control, $\left.{ }^{\sharp} \mathrm{P}<0.05\right) . \Delta \Psi \mathrm{m}$, mitochondrial membrane potential; con, control; DOX, doxorubicin; Ber, berberine.

pre-incubation with $1 \mu \mathrm{M}$ Ber markedly decreased the intensity of the DOX-induced rise in Rhod-2 fluorescence (Fig. 5A-b). Fig. 5B suggests that Rhod-2 fluorescence following $15 \mathrm{~min}$ of exposure to $1 \mu \mathrm{M}$ DOX was $303.2 \pm 65.1 \%$ of the baseline $(\mathrm{P}<0.001)$. Pre-incubation of $1 \mu \mathrm{M}$ Ber apparently lowered the $1 \mu \mathrm{M}$ DOX-induced rise in Rhod-2 fluorescence, which was reduced to $119.3 \pm 20.2 \%$ of the baseline $(\mathrm{P}<0.05)$. These data indicate that the application of Ber prior to DOX treatment prevented the elevation in mitochondrial $\mathrm{Ca}^{2+}$ overload induced by DOX alone, and enabled normal mitochondrial function.

\section{Discussion}

DOX is one of the most effective anti-tumour antibiotics, and novel methods to reduce or prevent the cardiotoxic side effects of DOX are expected. DOX-induced cardiotoxicity 


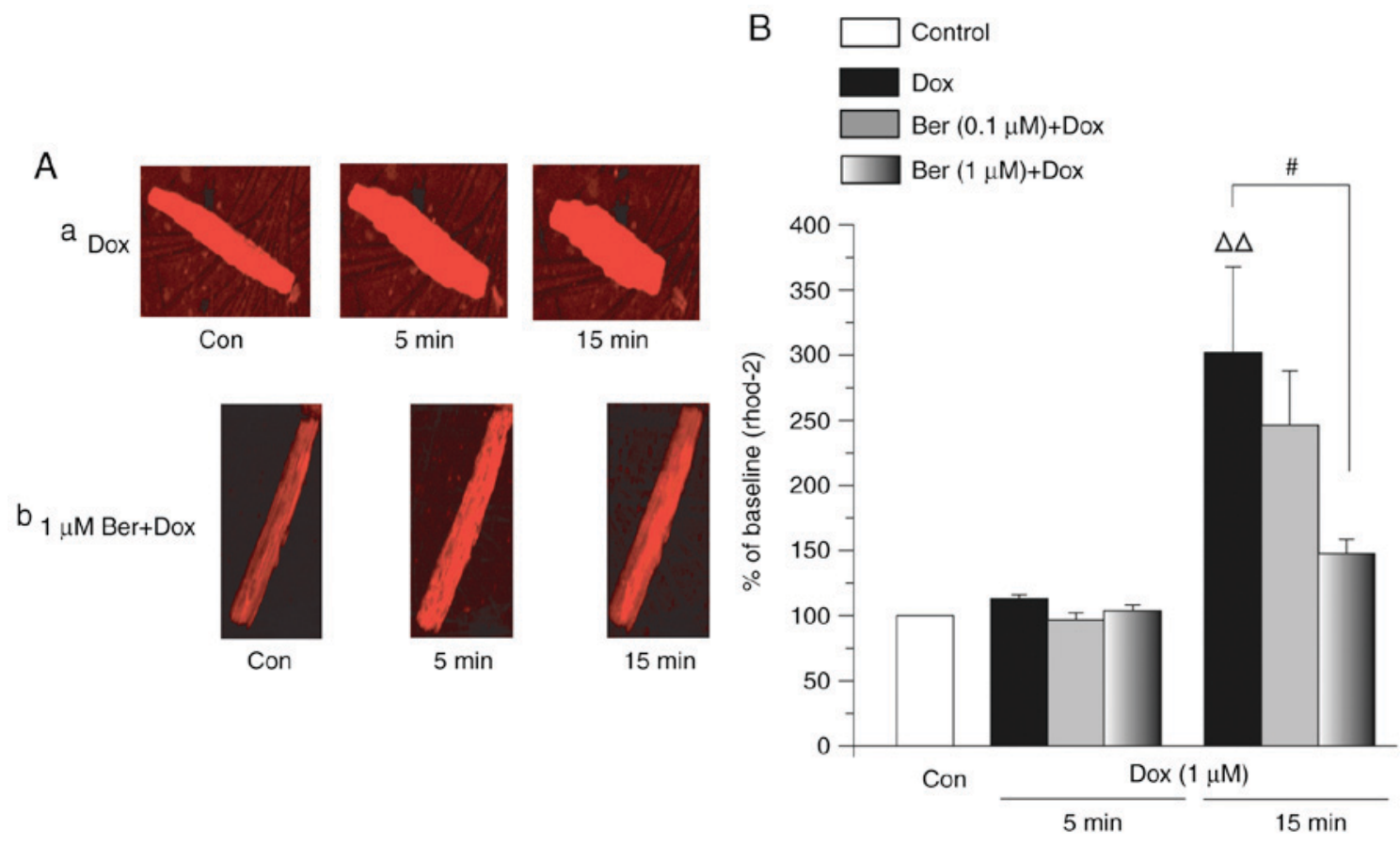

Figure 5. Effect of Ber on mitochondrial $\mathrm{Ca}^{2+}\left(\left[\mathrm{Ca}^{2+}\right]_{\mathrm{m}}\right)$ overload in Rhod 2-AM-loaded rat ventricular myocytes. (A) Confocal images of Rhod 2-AM fluorescence intensity from a single myocyte (magnification, $\mathrm{x} 40$ ). Cells were treated with DOX for 15 min as follows: (a) DOX-induced $\left[\mathrm{Ca}^{2+}\right]_{\mathrm{m}}$ elevation and (b) Effects of Ber $(1 \mu \mathrm{M})$ on $\left[\mathrm{Ca}^{2+}\right]_{\mathrm{m}}$ caused by DOX. (B) The effect of Ber on DOX-induced $\left[\mathrm{Ca}^{2+}\right]_{\mathrm{m}}$ overload at 5 min and 15 min on changes in relative Rhod 2-AM fluorescence intensity. ( $\mathrm{n}=6$ in each group) $\left({ }^{\Delta} \mathrm{P}<0.01\right.$ vs. control, $\left.{ }^{\mathrm{H}} \mathrm{P}<0.05\right)$. Con, control; DOX, doxorubicin; Ber, berberine; Rhod-2-AM, Rhod-2-acetoxymethyl; $\left[\mathrm{Ca}^{2+}\right]_{i}$, cytosolic calcium concentration.

manifests as acute effects (atrial and ventricular dysrhythmias) that vary from transient electrocardiographic abnormalities to dose-dependent cardiomyopathy and congestive heart failure $(15,16)$. A previous study of DOX cardiotoxicity demonstrated that the single high-dose model is widely used since it manifests certain characteristics of DOX-induced cardiotoxicity, which is equivalent to a single high-dose injection in patients with cancer (17).

Ber hydrochloride is an effective antioxidant and free radical scavenger that prevents ROS formation and exerts protective effects on cardiac, hepatic and renal functions $(18,19)$. Our previous study also demonstrated that Ber exhibited a protective effect on DOX-induced acute hepatorenal toxicity in rats (12). Whether Ber exerts a protective effect against DOX-induced cardiac injury is unknown. A number of cardioprotective agents, including dexrazoxane, amifostine and probucol, are of limited value in counteracting DOX cardiotoxicity and improving its clinical utility $(20,21)$. However, these scavengers exhibit clinical disadvantages, including a diminished anti-tumour effect and failure to protect the heart against DOX-induced injury in clinical settings. Tong et al (22) revealed that Ber treatment potentiated the sensitivity of cancer cells to DOX. Patil et al (23) also demonstrated that Ber suppressed tumour growth via the induction of apoptosis and cell cycle arrest in cancer cells. Taken together, based on these data, we hypothesized that combining DOX with Ber as a novel strategy for tumour therapy would not only increase the effect of DOX, but also prevent the cardiotoxicity induced by DOX. The present study investigated the mechanism of the protective effects of Ber against DOX-induced cardiotoxicity in rats.
A rat model of DOX-induced cardiotoxicity was developed in vivo and in vitro to determine the potential protective action of Ber against the cardiotoxic effects of DOX. The doses of Ber used in vivo were those that has exhibited the maximum cardio-protective effect in preliminary optimisation experiments (data not shown). Also, this result is consistent with a previous study that indicated that Ber demonstrated significant protective effects on heart tissue at $10 \mathrm{mg} / \mathrm{kg}$ in rats (24).

The data from the present study demonstrated that DOX administration was accompanied by a high mortality compared with the control group. Surviving animals suffered from an excessive degree of pericardial, pleural and peritoneal effusion, which indicated severe cardiac injury. The ability of Ber to protect against DOX-induced high mortality and effusion intensity score was considered an early sign of cardio-protection (25). Ber also attenuated DOX-induced histopathological and ultrastructural deteriorations. These data indicate that Ber is a potential protective agent against DOX injury. Oxidative damage and antioxidant deficiencies in cardiomyocytes are widely implicated as a primary cause for DOX-induced cardiac toxicity $(1,15)$, and these factors were utilized in the present study to examine the oxidant/antioxidant status of the rats. We used the single high-dose model and investigated whether Ber protected the heart from acute DOX toxicity. The current data demonstrated that cardiac and serum levels of MDA, CK and CK-MB were significantly elevated, and that the activities of the cardiac antioxidant enzymes CAT and SOD were significantly reduced, following DOX administration compared to the control group. These data clearly indicate a status of overt oxidative stress. Ber 
significantly decreased the DOX-associated elevation of serum MDA, CK and CK-MB activities, which are classical biomarkers of cardiotoxicity (4). It is well-known that the expression levels of B-type natriuretic peptide (BNP) and troponins are specific and sensitive indicators for cardiac damage (20). However, in the present study, severe cardiac damage induced by doxorubicin was observed, manifested by marked ascites and notable symptom of congestive heart failure. Since it was sufficient to analyse the cardiotoxicity by detecting $\mathrm{CK}$ and CK-MB levels in serum, it was not necessary to measure the expression levels of BNP and troponins. Nevertheless, means of measuring the expressions of BDP and troponins will be included in future studies, for full clarification of the protective mechanism of Ber on DOX-induced cardiotoxicity. The activities of CAT and SOD were significantly elevated in BER+DOX-treated groups compared with DOX-only administration in rats. These data are consistent with the current understanding of elevated cardiac lipid peroxidation accompanied by deteriorating antioxidant status was evident in the DOX-only treated rats. Ber pre-treatment significantly alleviated the oxidative stress in the DOX group, and Ber therapy completely prevented the biochemical and histopathological deteriorations caused by DOX These results suggest that Ber therapy during DOX treatment for cancer significantly protected the heart against DOX-induced injury. These data are in agreement with a study by Chen et al (12) and Hao et al (26). The initial damage of DOX-induced cardiotoxicity is likely oxidative in nature as it undergoes a one-electron reduction, resulting in the corresponding semiquinone, to form free radicals and superoxide radicals (27).

Oxidative damage to the cardiac mitochondria and cardiomyocyte is a cornerstone of DOX-induced cardiotoxicity, but a previous study suggested calcium overload as an additional important mechanism of DOX-induced cardiotoxicity (28). Previous studies have demonstrated that DOX opened sarcoplasmic reticulum calcium release channels and increased the maximal amount of calcium release $(29,30)$, inhibited $\mathrm{Na}^{+}-\mathrm{Ca}^{2+}$ exchange (31) or activated the L-type cardiac calcium channel (32). DOX may lead to calcium overload in cardiac cells, causing inadequate contraction and impairing mitochondrial calcium homeostasis, which alters energy metabolism and the generation of ROS (33) and may trigger the calcium-dependent mitochondrial permeability transition via opening of the permeability transition pores $(14,15)$. The opening of these pores induces the release of cytochrome $\mathrm{c}$, which is a critical step for apoptosis $(34,35)$. Mitochondria are key targets for anthracycline cardiotoxicity $(36,37)$, and may also be crucial for effective cardio-protection. The decrease in $\Delta \Psi_{\mathrm{m}}$ reduced the capacity for $\mathrm{Ca}^{2+}$ influx into mitochondria, as $\Delta \Psi_{\mathrm{m}}$ primarily drives mitochondrial calcium uptake (38). Therefore, the present study investigated intracellular calcium and mitochondrial calcium in cardiac myocytes following DOX treatment, and examined whether the protective effect of Ber on DOX-induced $\mathrm{Ca}^{2+}$ overload was associated with $\Delta \Psi_{\mathrm{m}}$ depolarization. The results demonstrated that the high local $\left[\mathrm{Ca}^{2+}\right]$ ${ }_{\mathrm{i}}$ level produced by DOX caused mitochondrial $\mathrm{Ca}^{2+}$ overload and a decrease in $\Delta \Psi_{\mathrm{m}}$, which may alter cardiac metabolism and lead to cardiomyocyte death. Incubation with Ber for
$1 \mathrm{~h}$ significantly reduced mitochondrial $\mathrm{Ca}^{2+}$ overload and significantly elevated cardiac energy metabolism. Ber treatment significantly suppressed the DOX-induced increase in $\left[\mathrm{Ca}^{2+}\right]_{\mathrm{i}}$. These results demonstrate that Ber inhibited the DOX-induced acute modifications in calcium homeostasis in cardiomyocytes, and suggest a protective role of Ber against DOX-induced cardiotoxicity. A previous study demonstrated that Ber may also attenuate DOX-induced cardiomyocyte apoptosis by inhibiting caspase-3 activation, adenosine 5'-monophosphate activated protein kinase $\alpha$ and tumour protein 53 phosphorylation (39). Ber is a potential candidate agent for co-administration with DOX to ameliorate its cardiotoxicity. Additional studies are required to thoroughly evaluate the potential protective effect of Ber in DOX-induced cardiotoxicity.

\section{Acknowledgements}

The authors would like to thank Professor Lian-Shan Zhang (Department of Pathology, Hebei Medical University, Shijiazhuang, China) for her help in histopathological analysis of the cardiac tissues.

\section{Funding}

The present study was supported by the Natural Science Foundation of China (grant nos. 81273600, 81302773 and 81773828) and the Natural Science Foundation of Hebei Province (grant nos. C2011206145, H2014206319 and H2018206297).

\section{Availability of data and materials}

The datasets used and/or analyzed during the current study are available from the corresponding author on reasonable request.

\section{Authors' contributions}

SWS and CX conceived and designed the study. YZ, CX, ZXW, YZW, XYC, HCG, KRX, KXW, and PJ performed the experiments. CX, YZ, YZW, and SWS wrote the paper. SWS and $C X$ reviewed and edited the manuscript. All authors read and approved the manuscript.

\section{Ethics approval and consent to participate}

All experiments were performed in compliance with the Guide for the Care and Use of Laboratory Animals and were reviewed and approved by the Ethics Committee for the Use of Experimental Animals at Hebei Medical University (Shijiazhuang, China).

\section{Consent for publication}

Not applicable.

\section{Competing interests}

The authors declare that they have no competing interests. 


\section{References}

1. Duggan ST and Keating GM: Pegylated liposomal doxorubicin: A review of its use in metastatic breast cancer, ovarian cancer, multiple myeloma and AIDS-related Kaposi's sarcoma. Drugs 71: 2531-2558, 2011.

2. Cortés-Funes $\mathrm{H}$ and Coronado $\mathrm{C}$ : Role of anthracyclines in the era of targeted therapy. Cardiovasc Toxicol 7: 56-60, 2007.

3. Minotti G, Menna P, Salvatorelli E, Cairo G and Gianni L: Anthracyclines: Molecular advances and pharmacologic developments in antitumor activity and cardiotoxicity. Pharmacol Rev 56: 185-229, 2004

4. Zhang YY, Yi M and Huang YP: Oxymatrine ameliorates doxorubicin-induced cardiotoxicity in rats. Cell Physiol Biochem 43. 626-635, 2017.

5. Bordoni A, Biagi P and Hrelia S: The impairment of essential fatty acid metabolism as a key factor in doxorubicin-induced damage in cultured rat cardiomyocytes. Biochim Biophys Acta 1440: 100-106, 1999.

6. Chatterjee K, Zhang J, Honbo N and Karliner JS: Doxorubicin cardiomyopathy. Cardiology 115: 155-162, 2010.

7. Christiansen S: Clinical management of doxorubicin-induced heart failure. J Cardiovasc Surg (Torino) 52: 133-138, 2011

8. Ludke AR, Al-Shudiefat AA, Dhingra S, Jassal DS and Singal PK: A concise description of cardioprotective strategies in doxorubicin-induced cardiotoxicity. Can J Physiol Pharmacol 87: 756-763, 2009.

9. Tan HL, Chan KG, Pusparajah P, Duangjai A, Saokaew S, Mehmood Khan T, Lee LH and Goh BH: Rhizoma coptidis: A potential cardiovascular protective agent. Front Pharmacol 7: 362.eCollection 2016,2016.

10. Tabeshpour J, Imenshahidi M and Hosseinzadeh H: A review of the effects of Berberis vulgaris and its major component, berberine, in metabolic syndrome. Iran J Basic Med Sci 20: $557-568,2017$

11. National Research Council (US) Committee for the Update of the Guide for the Care and Use of Laboratory Animals. Guide for the Care and Use of Laboratory Animals. 8th edition. Washington (DC): National Academies Press (US), 2011.

12. Chen X, Zhang Y, Zhu Z, Liu H, Guo H, Xiong C, Xie K, Zhang X and Su S: Protective effect of berberine on doxorubicin induced acute hepatorenal toxicity in rats. Mol Med Rep 13: 3953-3960, 2016.

13. Kelishomi RB, Ejtemaeemehr S, Tavangar SM, Rahimian R, Mobarakeh JI and Dehpour AR: Morphine is protective against doxorubicin-induced cardiotoxicity in rat. Toxicology 243 96-104, 2008.

14. Xiong C,Li JX, Guo HC, Zhang LN, Guo W, Meng J and Wang YL: The $\mathrm{H}_{1}-\mathrm{H}_{2}$ domain of the $\alpha 1$ isoform of $\mathrm{Na}+\mathrm{K}+-\mathrm{ATPase}$ is involved in ouabain toxicity in rat ventricular myocytes. Toxicol Appl Pharmacol 262: 32-42, 2012.

15. Asensio-López MC, Soler F, Pascual-Figal D, Fernández-Belda F and Lax A: Doxorubicin-induced oxidative stress: The protective effect of nicorandil on HL-1 cardiomyocytes. PLoS One 12 e0172803, 2017.

16. Jain D, Ahmad T, Cairo M and Aronow W: Cardiotoxicity of cancer chemotherapy: Identification, prevention and treatment. Ann Transl Med 5: 348, 2017.

17. Li T, Danelisen I and Singal PK: Early changes in myocardial antioxidant enzymes in rats treated with adriamycin. Mol Cell Biochem 232: 19-26, 2002.

18. Lee IA,Hyun YJ and Kim DH: Berberine ameliorates TNBS-induced colitis by inhibiting lipid peroxidation, enterobacterial growth and NF-кB activation. Eur J Pharmacol 648: 162-170, 2010.

19. Li P, Ren J, Duan C, Lin C and Liu J: Effects of four components of Rhizoma Corydalis on anoxia and peroxidation injuries in neonatal cardiomyocytes. Zhongguo Zhong Yao Za Zhi 35: 84-88, 2010 (In Chinese)

20. Che FF, Liu Y and Xu CG: Schisandrin B prevents doxorubicin-induced cardiotoxicity in rabbits. Sichuan Da Xue Xue Bao Yi Xue Ban 41: 24-28, 2010 (In Chinese).

21. Li T and Singal PK: Adriamycin-induced early changes in myocardial antioxidant enzymes and their modulation by probucol. Circulation 102: 2105-2110, 2000.
22. Tong N, Zhang J, Chen Y, Li Z, Luo Y, Zuo H and Zhao X: Berberine sensitizes mutliple human cancer cells to the anticancer effects of doxorubicin in vitro. Oncol Lett 3: 1263-1267, 2012.

23. Patil JB, Kim J and Jayaprakasha GK: Berberine induces apoptosis in breast cancer cells (MCF-7) through mitochondrial-dependent pathway. Eur J Pharmacol 645: 70-78, 2010.

24. Zhang YJ, Yang SH, Li MH, Iqbal J, Bourantas CV, Mi QY, Yu YH, Li JJ, Zhao SL, Tian NL and Chen SL: Berberine attenuates adverse left ventricular remodeling and cardiac dysfunction after acute myocardial infarction in rats: Role of autophagy. Clin Exp Pharmacol Physiol 41: 995-1002, 2014

25. Takemura G and Fujiwara H: Doxorubicin-induced cardiomyopathy from the cardiotoxic mechanisms to management. Prog Cardiovasc Dis 49: 330-352, 2007.

26. Hao G, Yu Y, Gu B, Xing Y and Xue M: Protective effects of berberine against doxorubicin-induced cardiotoxicity in rats by inhibiting metabolism of doxorubicin. Xenobiotica 45: 1024-1029, 2015.

27. Wang S, Konorev EA, Kotamraju S, Joseph J, Kalivendi S and Kalyanaraman B: Doxorubicin induces apoptosis in normal and tumor cells via distinctly different mechanisms. Intermediacy of $\mathrm{H}(2) \mathrm{O}(2)$ - and p53-dependent pathways. J Biol Chem 279: 25535-25543, 2004.

28. Solem LE, Henry TR and Wallace KB: Disruption of mitochondrial calcium homeostasis following chronic doxorubicin administration. Toxicol Appl Pharmacol 129: 214-222, 1994.

29. Zorzato F, Sarviati G, Facchinetti T and Volpe P: Doxorubicin induces calcium release from terminal cisternae of skeletal muscle. A study on isolated sarcoplasmic reticulum and chemically skinned fibers. J Biol Chem 260: 7349-7355, 1985.

30. Kim DH, Landry AB III, Lee YS and Katz AM: Doxorubicin-induced calcium release from sarcoplasmic reticulum vesicles. J Moll Cell Cardiol 21: 433-436, 1989.

31. Caroni P, Villani F and Carafoli E: The cardiotoxic antibiotic doxorubicin inhibits the $\mathrm{Na}+\mathrm{Ca} 2+$ exchange of dog heart sarcolemmal vesicles. FEBS Lett 130: 184-186, 1981.

32. Keung EC, Toll L, Ellis M and Jensen RA: L-type cardiac calcium channels in doxorubicin cardiomyopathy in rats morphological, biochemical, and functional correlations. J Clin Invest 87: 2108-2113, 1991.

33. Przygodzki T, Sokal A and Bryszewska M: Calcium ionophore A23187 action on cardiac myocytes is accompanied by enhanced production of reactive oxygen species. Biochim Biophys Acta 1740: 481-488, 2005.

34. Petrosillo G, Ruggiero FM, Pistolese M and Paradies G: $\mathrm{Ca} 2+-$ induced reactive oxygen species production promotes cytochrome $\mathrm{c}$ release from rat liver mitochondria via mitochondrial permeability transition (MPT)-dependent and MPT-independent mechanisms: Role of cardiolipin. J Biol Chem 279: 53103-53108, 2004.

35. Waring P: Redox active calcium ion channels and cell death. Arch Biochem Biophys 434: 33-42, 2005.

36. Li L, Takemura G, Li Y, Miyata S, Esaki M,Okada H, Kanamori H, Khai NC, Maruyama R, Ogino A, et al: Preventive effect of erythropoietin on cardiac dysfunction in Doxorubicin-induced cardiomyopathy. Circulation 113: 535-543, 2006.

37. Yeh YC, Lai HC, Ting CT, Lee WL, Wang LC, Wang KY, Lai HC and Liu TJ: Protection by doxycycline against doxorubicin-induced oxidative stress and apoptosis in mouse testes. Biochem Pharmacol 74: 969-980, 2007.

38. Rahn CA, Bombick DW and Doolittle DJ: Assessment of mitochondrial membrane potential as an indicator of cytotoxicity. Fundam Appl Toxicol 16: 435-448, 1991.

39. Lv X, Yu X, Wang Y, Wang F, Li H, Wang Y, Lu D, Qi R and Wang $\mathrm{H}$ : Berberine inhibits doxorubicin-triggered cardiomyocyte apoptosis via attenuating mitochondrial dysfunction and increasing Bcl-2 expression. PLoS One 7: e47351, 2012.

(i) $\odot$ This work is licensed under a Creative Commons Attribution-NonCommercial-NoDerivatives 4.0 International (CC BY-NC-ND 4.0) License. 